\title{
Use case 3: post accidental site remediation - CEA
}

\author{
Yvon Desnoyers $^{1, *}$, Claire Faucheux ${ }^{1}$, and Nadia Pérot ${ }^{2}$ \\ ${ }^{1}$ Geovariances, 49bis avenue Franklin Roosevelt, 77210 Avon, France \\ 2 DEN/CAD/DER/SESI/LEMS, CEA Cadarache, 13108 Saint Paul-Lez-Durance, France
}

Received: 23 October 2019 / Received in final form: 13 November 2019 / Accepted: 4 December 2019

\begin{abstract}
Within the H2020 INSIDER project, the main objective of work package 3 (WP3) is to draft a sampling guide for initial nuclear site characterization in constraint environments, before decommissioning, based on a statistical approach. This paper is dedicated to the sampling strategy for use case 3 (UC3) about contaminated soils, in the context of post-incidental remediation of a site. For this use case, the constraint environment comes from the difficulty to collect samples beneath a building on the one hand and the fact that samples were collected in the past with no possibility for additional samples. This task has been initiated by gathering prior knowledge for the contaminated site and analysing the available dataset (historical assessment + available data from non-destructive and destructive analyses).
\end{abstract}

\section{Introduction}

The EURATOM work program project INSIDER was launched in June 2017 (18 partners from 10 European countries). It aims at improving the management of contaminated materials arising from decommissioning and dismantling (D\&D) operations by proposing an integrated methodology of characterization. The methodology is based on advanced statistical processing and modelling, coupled with adapted and innovative analytical and measurement methods, in line with sustainability and economic objectives.

An integrated and overall approach of pre-decommissioning characterization consists in evaluating historical data, making on-site measurement campaigns, sampling and analysing, developing scaling factors and applying numerical codes. The final objective of work package 3 is to draft a guideline for sampling in the field of initial nuclear site characterisation in constraint environments in view of decommissioning, based on a statistical approach. In order to reach this goal, a first review of available and relevant standards, guides and methods used for sampling design and data analysis has been initially completed [1]. Therefore, statistical approaches to be used in constraint environment have been described as a generic strategy for handling problem definition, data analysis and sampling design definition [2]. This second task has then been implemented in a web-based application presenting the strategy in a more user-friendly way.

\footnotetext{
* e-mail: desnoyers@geovariances.com
}

Within task 3.3, the strategy is thoroughly tested in practice within three different test cases (this task has been initiated by gathering prior knowledge for each test case (historical assessment + available data from non-destructive and destructive analyses) and developing the different sampling plans):

- use case 1: Decommissioning of a back/end fuel cycle and/or research facility: Radioactive liquid and sludge in tank at JRC Ispra (Italy);

- use case 2: Decommissioning of a nuclear reactor: Activated bio-shield concrete of the BR3 reactor (Belgium);

- use case 3: Post accidental land remediation: Contaminated soils beneath a CEA building (France).

The return-of-experience will allow refining the overall approach for the final guideline within the INSIDER project.

This paper is precisely dedicated to the sampling strategy for use case 3 (UC3) about contaminated soils, in the context of post-incidental remediation of a site [3]. For this use case, the constraint environment comes from the difficulty to collect samples beneath a building on the one hand and the fact that samples were collected in the past with no possibility for additional samples. However, new measurements (both non-destructive and destructive) are possible on the existing samples, if appropriate and relevant for the overall characterization of this site. This may be performed in order to improve the uncertainty quantification within the INSIDER project at WP6 level (Performance analysis and overall uncertainty), combining outputs from WP3 (sampling strategy), WP4 (In lab analysis) and WP5 (on site measurements). 


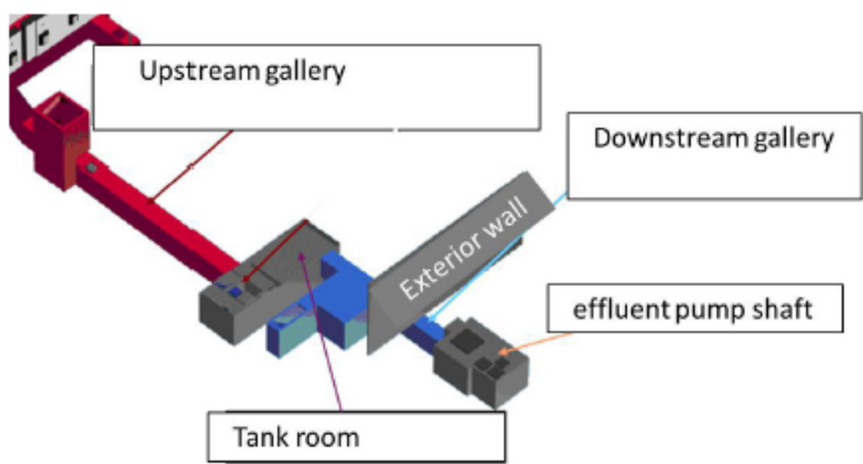

Fig. 1. Use case 3 location beneath a tank room of a former nuclear facility.

\section{Presentation of UC3: contaminated soils beneath a CEA building (France)}

For some confidentiality reasons the strict minimum of the site context information has been made available within the INSIDER project. However, this situation is sometimes representative of real circumstances for old facilities for instance, for which historical knowledge results are very limited. What can be mentioned is this nuclear facility was devoted to radiochemistry on transuranium elements. It was under operation until 1992 on a CEA site in France. A tank room is located beneath the basement and collected various effluents from experiments through pipes along the upstream gallery. An additional downstream gallery leads to the lowest point of the facility (effluent pump shaft). The main contaminated soil area is located beneath this building, just below the former tank room (Fig. 1).

It has been reported that different incidents occurred during nuclear facility operation. Contamination of soils beneath the tank room with few $\mathrm{TBq}$ of various alpha et beta emitters is expected due to leaks of very high radioactive effluent in the tank room and several potential contamination pathways to reach the soils such as ingress, cracks or expansion joints of the concrete slab.

In order to improve the radiological assessment of soils below this tank room, different sampling campaigns have been conducted. They consist in 7 horizontal drill holes between 2012 and 2015 . The 7 drill holes are distributed in two horizontal layers at 0.5 and $1.5 \mathrm{~m}$ below bottom of the tank room slab (Fig. 2). The drilling machine was located at the bottom of a circular pit outside of the building to access this area and go horizontally. This approach is not very common for soil sampling as generally vertical drill holes from the top surface is preferred. However, due to the presence of the building and the impossibility to introduce the drilling machine in the tank room, this deported and long-range horizontal drilling location has been preferred.

Collected soil samples are quite comparable with the presence of small to intermediate size gravel in a sandy matrix. Samples have been then constituted taking 500$800 \mathrm{~g}$ mass (geometry standardized at $500 \mathrm{~mL}$ ) assuming two core lengths: 0.5 or $1 \mathrm{~m}$. First observations of radioactivity distribution depend on soil compositions and radionuclides chemical properties. In addition, activity seems to be concentrated in small soil particles.

Within the INSIDER project, the existing dataset is considered as the final one for this use case 3 . There is no sampling design definition as for the other use cases, but the statistical analysis promises to bring interesting and relevant conclusions for the whole project due to the number (220) of available samples. This will be performed by conducting sensitivity analysis (extracting different sub-datasets) and possibly valuate the correlation with 5 -cm gamma-scanning values along the cores.

\section{Characterisation objectives}

\subsection{Global estimation of statistical quantities}

For the preparation and management of a soil remediation project, some global quantities need to be estimated in a sound way. For instance, average activity concentration value for the whole area (as well as its related uncertainty) is an interesting parameter and needs to be statistically estimated. The different statistical tests and inequalities can be derived if the dataset is compatible with the underlying hypotheses. Spatial and/or statistical biases need to be carefully addressed. Consequently, the total activity (still along with its confidence level) can be estimated as an accumulation (knowing the total volume and the matrix density).

Other global statistical quantities are linked to specific values. At that stage, global estimations of volumes exceeding radiological thresholds significantly help for the classification according to the different waste categories.

\subsection{D distribution map of activity concentration and waste segregation}

In addition to the global estimates presented previously, some local estimates are very relevant for the adequate management of remediation projects. The analysis of depth profiles combined with the horizontal distribution leads to $2 \mathrm{D} / 3 \mathrm{D}$ representations depending on the dataset spatial organization. Appropriate geostatistical methods need to be carefully selected given the spatial continuity of the phenomenon and the database configuration (linearity, stationarity, multivariate, trend, etc.).

Local estimates derive from comparison to specific radiological values. The local probability of exceeding a radiological threshold then leads to the volumes to be excavated in the different waste categories. At this stage, radiological thresholds for the different waste categories are not yet fully defined, for site release values particularly (output of operator impact assessment 


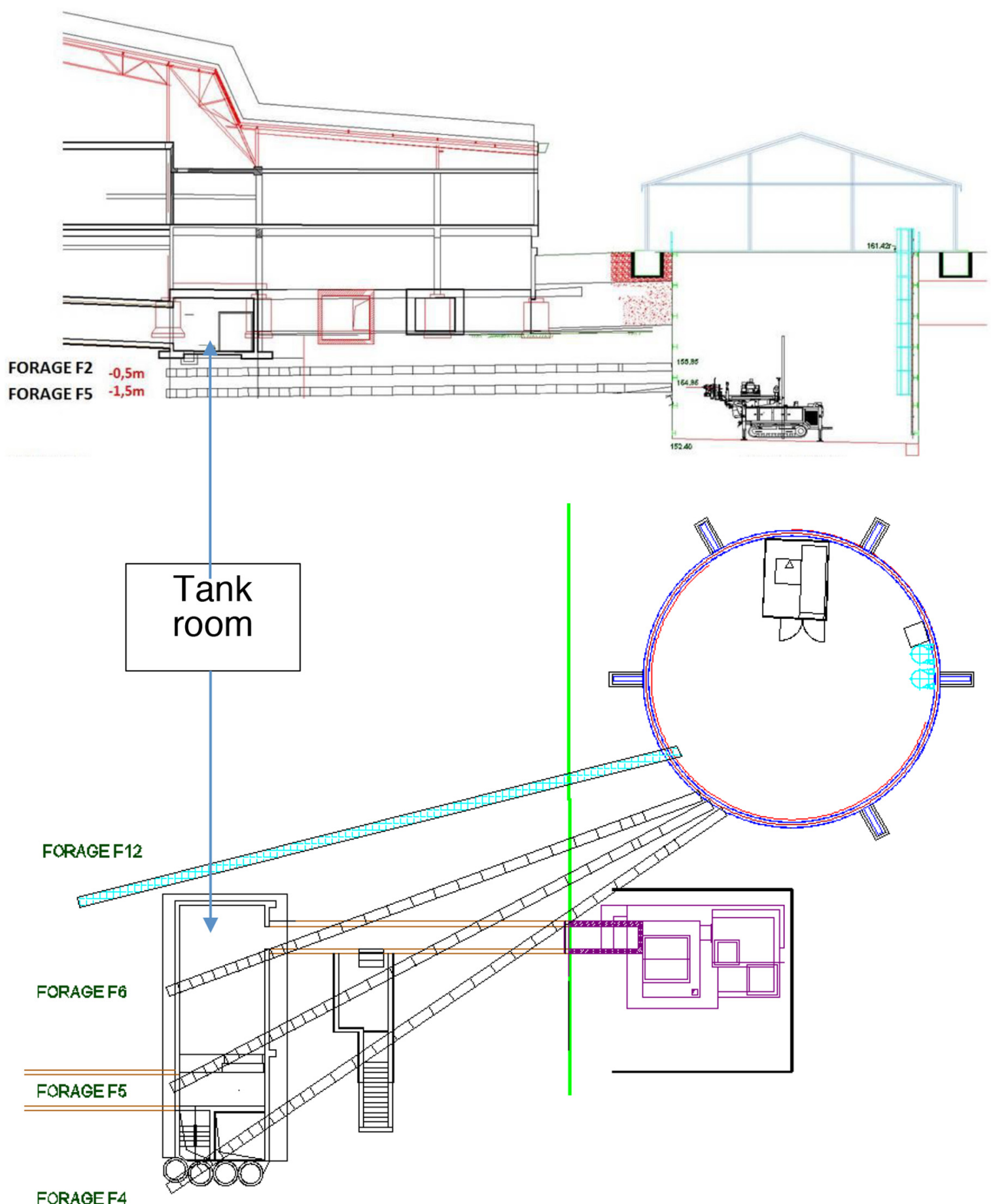

Fig. 2. Vertical cross section (top) of the horizontal drilling layers. Top view (bottom) of the angular distribution of drill holes at depth $-1.5 \mathrm{~m}$.

study). For the segregation between Very-Low Level waste and Low-Level waste, as a French site, ANDRA (National Radioactive Waste Management Agency) specifications are quite clear and require a weighted sum calculation according to scaling factor and nuclide class (IRAS). In any case we will need to have/apply a certain radionuclide vector (including uncertainties) that must be determined and applied as well. These aspects will need a lot of attention in the coming analysis.

This classification decision can also take the remediation support into account (e.g. averaging out over $1 \mathrm{~m}^{3}$ or 1 ton or other values). All classification decisions then require working on estimation uncertainties. 

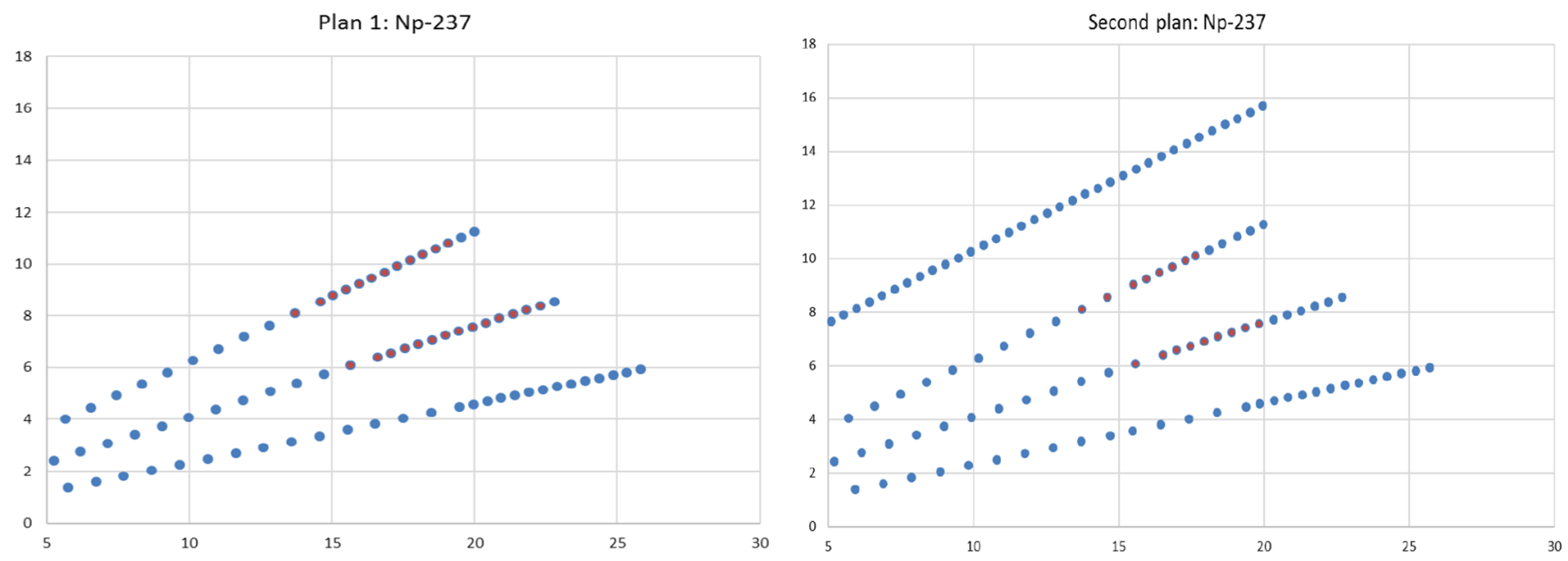

Fig. 3. Base maps of the two sampled layers (above detection limit in red). Drillings at depth $-0.5 \mathrm{~m}$ on the left and at $-1.5 \mathrm{~m}$ on the right.

Finally, the different costs for the soil excavation, waste confection and disposal may be integrated as a forecast of the remediation project and compared and balanced to the initial characterization costs.

\section{Ongoing works}

\subsection{Dealing with a unique existing dataset}

As there is no possibility for new samples and new in situ measurements, the existing dataset is considered as the final one within the INSIDER project for this use case 3 . However, non-destructive measurements and new samples can be performed on the existing cores.

There is no sampling design definition as for the other use cases, but the statistical analysis promises to bring interesting and relevant conclusions for the whole INSIDER project due to the number of available samples (see also discussion in Sect. 4.4).

In addition, sensitivity to dataset extension will be studied as 2 zones can be identified: the inner area with the highest activity levels (corresponding to the $50 \mathrm{~cm}$ sampling resolution along the cores) and the accessibility area (with a $1 \mathrm{~m}$ mesh). Statistical outputs will differ because of this spatial delineation.

As it seems to be a relevant 3D contamination, areas with high estimation uncertainties as well as extrapolation areas will finally be identified. They would have been used for recommendations of new samples in the case of a site with possible additional investigation.

\subsection{Preliminary analysis}

Looking to the spatial organisation, samples were collected along 7 cores, distributed in two layers. The sampling resolution is $1 \mathrm{~m}$ and is refined to $50 \mathrm{~cm}$ in the interest area (Fig. 3). Therefore, the first 10-15 meters from the origin point can be considered as an accessibility distance. The last borehole (named F12) is different as it presents a $50 \mathrm{~cm}$ sampling resolution from the beginning. The total number of analysed samples then reaches 220, which can be considered as a large dataset at first glance.

Preliminary direct measurements have been performed along the cores. They consist in gamma and $\mathrm{X}$ scanning (gross counting) on a regular $5 \mathrm{~cm}$ mesh. These indirect measurements probably serve as a semi-quantitative characterisation of the gamma content of soil samples. However, these measurements are not part of the transmitted data but the operator has agreed to add them to this use case. That would be very relevant for INSIDER project to be able to combine in-situ and destructive values in order to improve the estimations (and reduce the uncertainties).

Main nuclides for laboratory analyses are Np-237, Pa233 for gamma emitters and Sr-90 for beta emitter. Other gamma emitters such as Co-60, Eu-152, Uranium and Thorium chains are not present (or at very low levels, close to detection limits). Cs-137 and Am-241 are detected on some samples. In addition, the different Plutonium isotopes $(238,239+240$ and 241) have been quantified using appropriate alpha measurements. It seems that alpha spectrometry (dissolution, extraction, electrodeposition) and beta counting (liquid scintillation with a detection limit of $30 \mathrm{~Bq} / \mathrm{kg}$ ) have been initially decided on the basis of the higher dose rate location on the core. At the end, most of all samples have been measured for alpha emitters.

As for statistical distribution, as generally observed for soil contamination, the histogram is very skewed (as presented on Fig. 4 for Np-237) with a lot of measurements at detection limit and with intermediate to high value in a more limited number.

In addition, a correlation analysis puts into evidence some interesting statistical links between nuclides except for Cs-137, Co-60 and partly Plutonium isotopes. The correlation matrix at Figure 5 then presents linear regression coefficients on the bottom left part and corresponding correlation dispersion shape on the upper right part (the diagonal of the nuclides has been reordered to underline correlated groups). Correlation values are all positive from very low values (close to 0 or statistically 
insignificant) to 1 for perfect positive correlation. This analysis also identifies a few outliers that are under investigation and verification to correct them if they are erroneous values (mistyping for instance) or if they have to be considered as singular values.

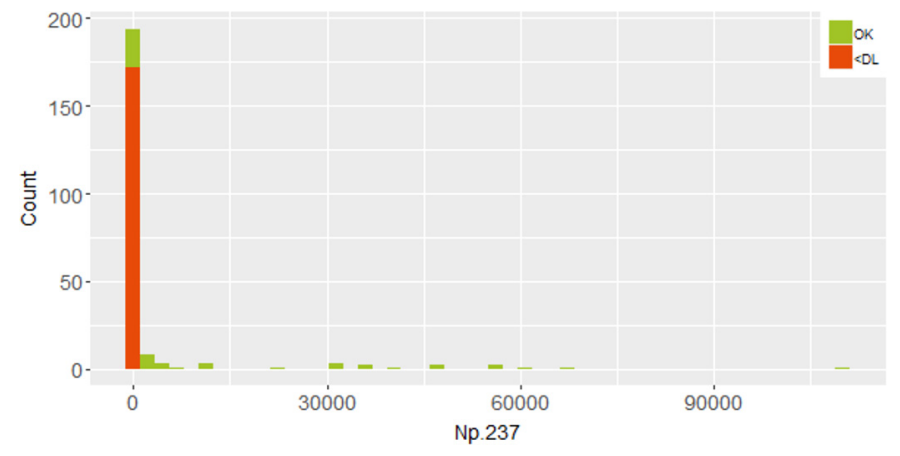

Fig. 4. Histogram of Np-237 activity levels.

\subsection{First geostatistics results}

A sound geostatistics processing of the data always starts with a deep preliminary data analysis. This pre-processing step is crucial to build a consistent database. First, spatial bias due to non-probabilistic sampling is tackled with declustering techniques (spatial weighting). The joint analysis of the statistical distribution (histogram) may identify heterogeneous populations (spatial, temporal, units, sample support, etc.) that need to be corrected or separated for a proper understanding. A skewed distribution requires a non-linear data transformation (indicator or logarithm eventually but more interestingly Gaussian anamorphosis as shown on Fig. 6). In the presence of a correlated variable, a multivariate processing can be implemented at all stages, and is very useful if the auxiliary data is denser than that of the primary variable (that points is not addressed yet with the gamma scanning values).

The heart of geostatistics is the analysis and the modelling of the spatial continuity using the variogram.

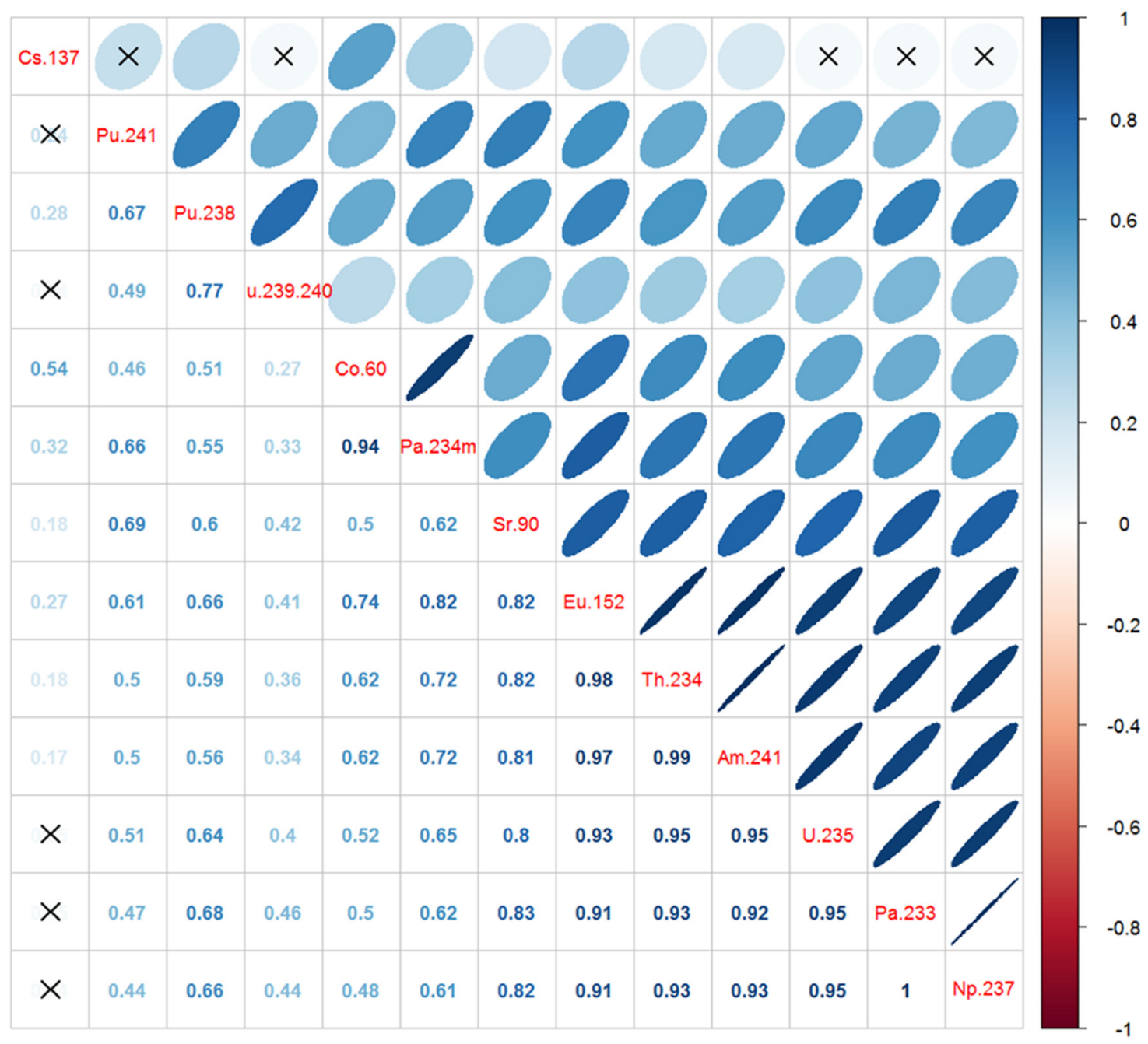

Fig. 5. Correlation matrix between nuclides. 


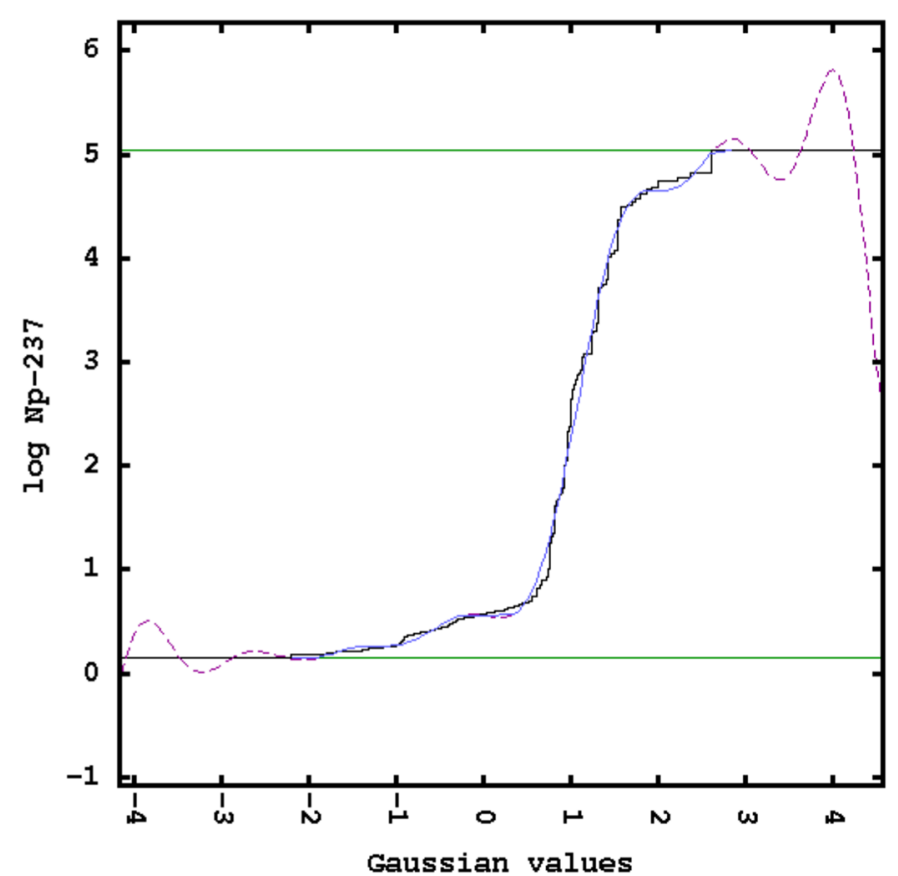

Fig. 6. Gaussian anamorphosis of the data logarithm for Np-237.

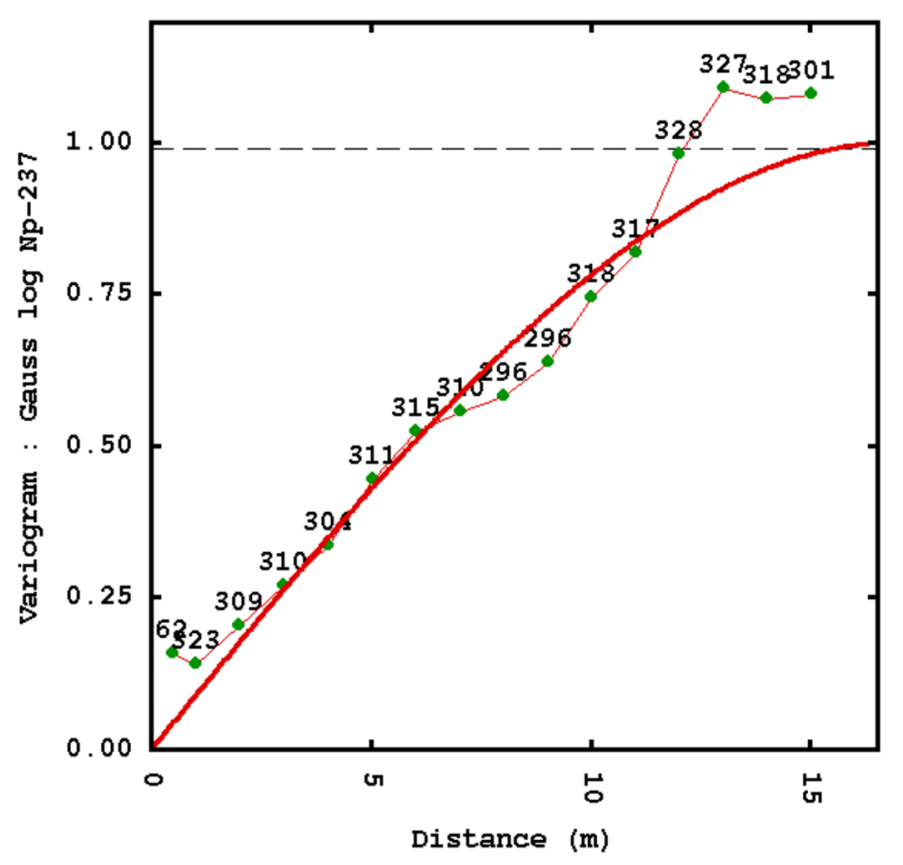

Fig. 7. Horizontal variogram for $\mathrm{Np}-237$.
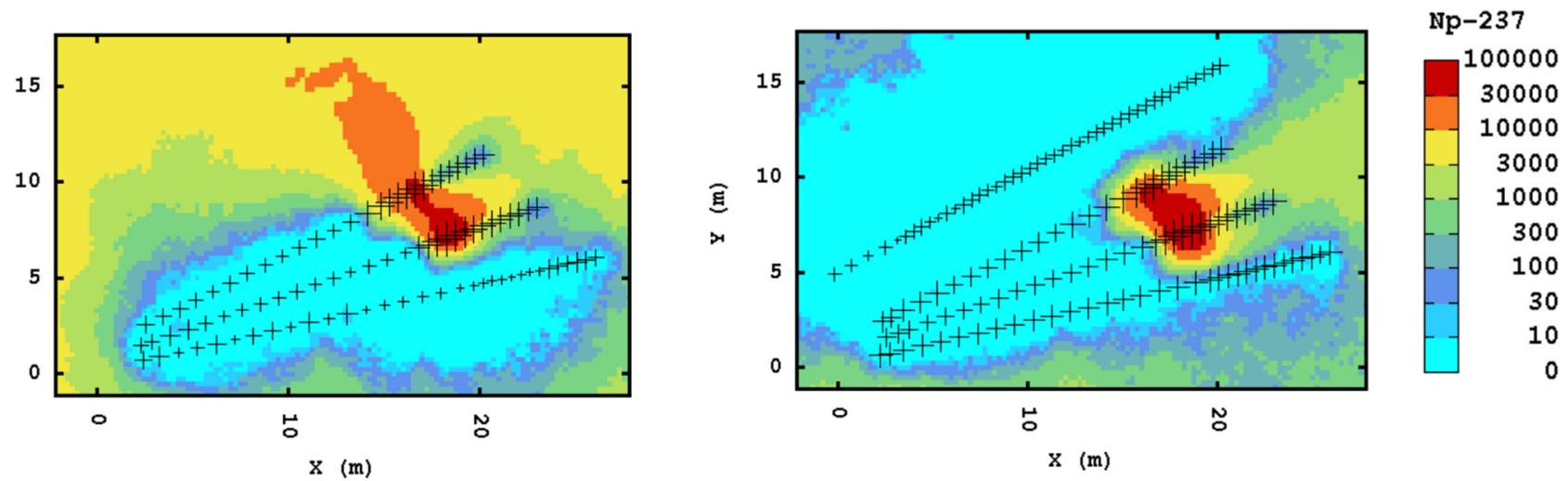

Fig. 8. Interpolation maps for Np-237 for the two layers using simulation mean.
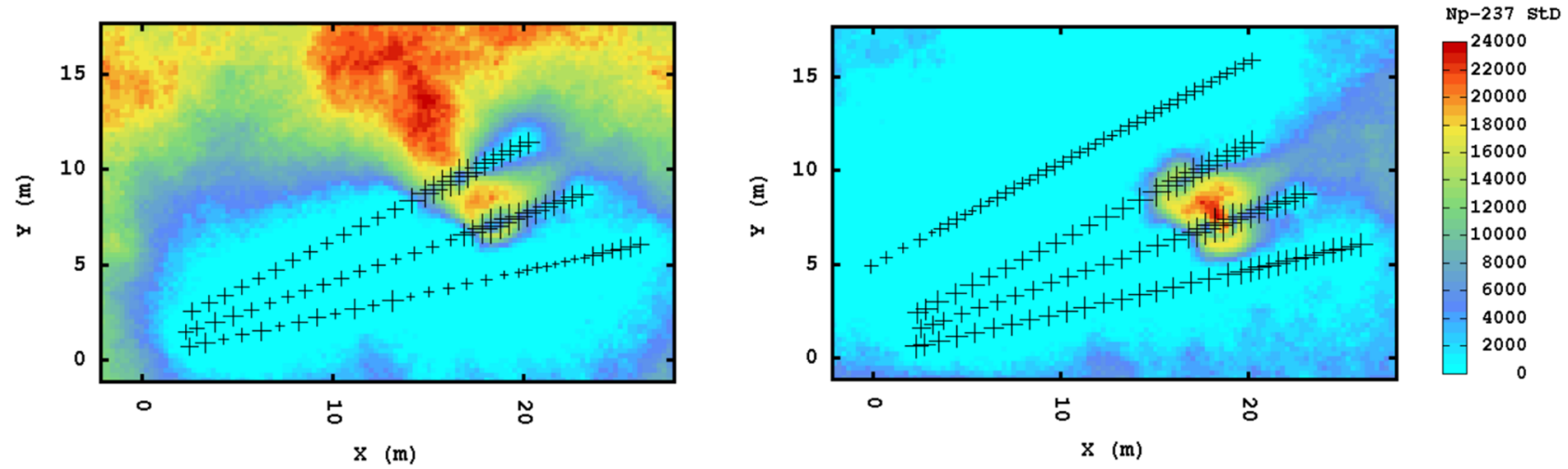

Fig. 9. Estimation uncertainty for Np-237 as standard deviation of simulations. 

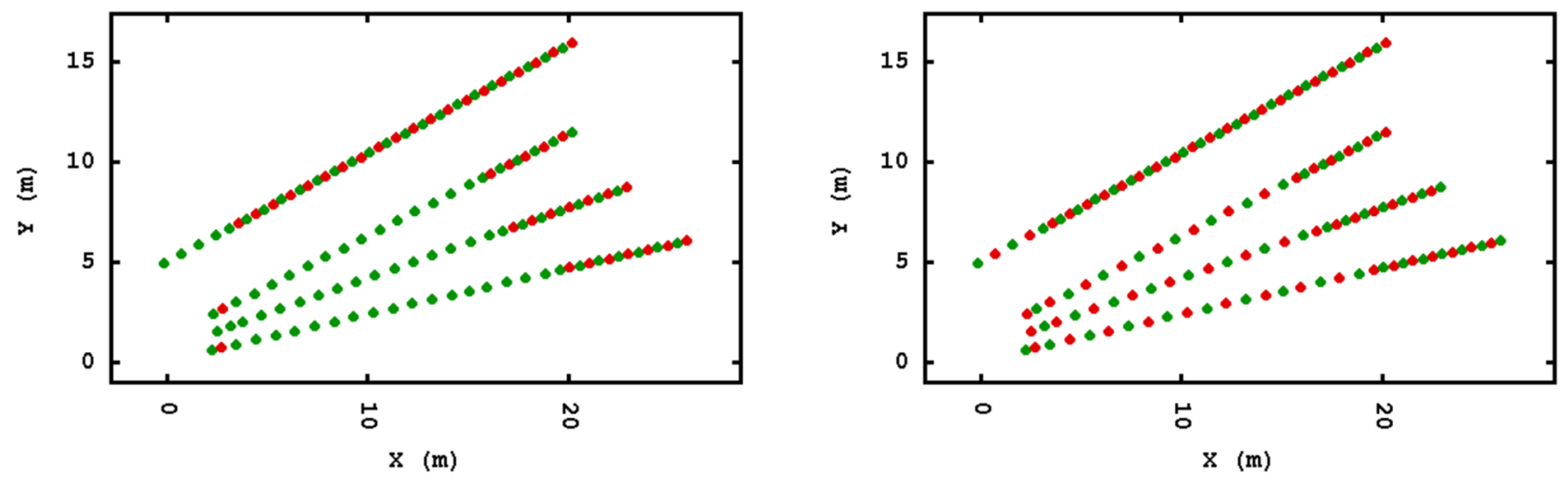

Fig. 10. Examples of sampling reduction ( $1 \mathrm{~m}$ on the left, 1 sample out of 2 on the right).

Anisotropy is generally relevant in $3 \mathrm{D}$, and also in $2 \mathrm{D}$ if there is a specific direction for the phenomenon. In the present case, the vertical continuity can only be estimated at $1 \mathrm{~m}$ distance due to the specific two-layer configuration. In the horizontal plane, the variographic analysis makes the difference between the variability along a drill-hole and between samples from different drill-holes. Only the resulting and anisotropic variogram is presented on Figure 7 . It shows a very clear spatial structure with no nugget effect (variability at small scale) and a linear behaviour at the site scale.

The first output of geostatistics is the interpolation, or the kriging estimate. At that stage, only punctual estimates are presented but block estimates are expected to be realized. In addition, the estimation uncertainty is also presented as it is fully part of the estimation.

Future works will deal with non-linear estimates. Risk of exceeding a threshold with prior Gaussian anamorphosis (conditional expectation) is specifically relevant for radiological waste classification. Another approach is to compute conditional simulations (which can be seen as spatially consistent Monte-Carlo simulations). Post-processing of these simulations enables regular (grid) and irregular (polygon) change-of-support modelling (averaging on a larger support) as well as any statistics: mean (Fig. 8), variance (Fig. 9), probability of exceeding a threshold. Global estimates (volumes according to a threshold, source term) are obtained in the same way.

\subsection{Sensitivity analysis by data reduction}

Despite the dataset limitations for the definition of the sampling plan as described before, the large number of samples enables a sound sensitivity analysis. Sub-dataset will be extracted from the full dataset in order to quantify the impact on the estimates and their related uncertainties. Different possibilities can be imagined:

- Reduction of sample number per drill hole, as presented on Figure 10.

- Reduction of drill hole number.

- Integrating correlation between nuclides by reducing some laboratory analyses.
This approach will definitely provide interesting outputs for the INSIDER project as for impact on sampling strategy (in particular sampling density).

\section{Conclusion}

In WP3 of the H2020 INSIDER project, the third task is the implementation of the sampling strategy and data analysis on three use cases in the field of initial nuclear site characterization. This approach is currently and thoroughly tested in practice within different use cases: radioactive liquids in tanks, activated concrete, contaminated soils.

For this third use case (UC3), the characterisation of contaminated soils beneath a nuclear building started with the sampling campaign several years ago. The specific work within the INSIDER project is to apply the described methodology (statistics, geostatistics) on the existing dataset with the possibility to quantify the impact of sampling reduction on the estimation uncertainty. Several indicators will be used to present the results. In particular, the waste volumes will be compared for two classification thresholds (very low level waste and low level waste).

The return-of-experience will allow refining the overall methodology for the final guideline developed within INSIDER WP3, describing the statistical approach and taking the uncertainty budget into consideration, potentially allowing further refinement of the web-based application in the final stage.

The INSIDER project received funding from the Euratom Research and Training Programme 2014-2018 under grant agreement No 755554 .

\section{Author contribution statement}

The task leader of this study within the INSIDER project is Yvon Desnoyers. He is also the main contributor to the article writing. Claire Faucheux was mainly involved in the computation part, performing the data preparation, analysis, modelling and mapping using Isatis software. Nadia Pérot contributed to this work as representative of CEA, owner and provider of the dataset, with also critical and expert judgement and by providing writing support and proofreading. 


\section{References}

1. N. Pérot, Y. Desnoyers, G. Augé, F. Aspe, S. Boden, B. Rogiers, O. Sevbo, O. Nitsche, INSIDER WP3-Sampling strategy - Report on the state of the art, Deliverable 3.1, 2017
2. B. Rogiers, S. Boden, N. Perot, Y. Desnoyers, O. Sevbo, O. Nitzsche, INSIDER WP3-Sampling strategy - Report on statistical approach, Deliverable D3.2, 2018

3. Y. Desnoyers, N. Pérot, INSIDER WP3-Sampling strategy Sampling plan for use case 3: Contaminated soils, Deliverable D3.6, 2019

Cite this article as: Yvon Desnoyers, Claire Faucheux, Nadia Pérot, Use case 3: post accidental site remediation - CEA, EPJ Nuclear Sci. Technol. 6, 13 (2020) 